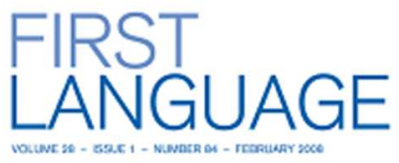

\title{
Asking for Action or Information? Crosslinguistic Comparison of Interrogative Functions in Early Child Cantonese and Mandarin
}

\begin{tabular}{|r|l|}
\hline Journal: & First Language \\
\hline Manuscript ID: & Draft \\
\hline Kanuscript Type: & Original Manuscript \\
\hline Keywords: & $\begin{array}{l}\text { Interrogative function, early child Mandarin, early child Cantonese, corpus- } \\
\text { based study, cross-linguistic study }\end{array}$ \\
\hline Abstract: & $\begin{array}{l}\text { Request for information (RfI) is believed to be the universally dominant } \\
\text { function of young children's questioning, whereas request for action (RfA) } \\
\text { has been reported to be the leading interrogative form used in early child } \\
\text { Cantonese. The possibility of crosslinguistic variability prompts further } \\
\text { research and comparison with additional languages. This study compared } \\
\text { the interrogatives elicited from two early Chinese language corpora: Early } \\
\text { Childhood Cantonese Corpus (ECCC) and Early Childhood Mandarin } \\
\text { (ECMC). Altogether, 1,214 and 942 question types were elicited from ECCC } \\
\text { and ECMC, respectively. Analyses indicated that: (1) All the interrogative } \\
\text { functions identified in an earlier study of Cantonese were also observed in } \\
\text { the early Mandarin interrrogatives; and (2) both RfA (49.9\%) and RfI } \\
\text { (45.5\%) were the most frequently observed functions of early child } \\
\text { Chinese interrogatives. This crosslinguistic evidence suggests that follow- } \\
\text { up studies are needed to further explore the possible influences of } \\
\text { language, culture and communication tasks on children's uses of } \\
\text { interrogative forms. }\end{array}$ \\
\hline
\end{tabular}

\section{SCHOLARONE ${ }^{\text {m }}$ \\ Manuscripts}




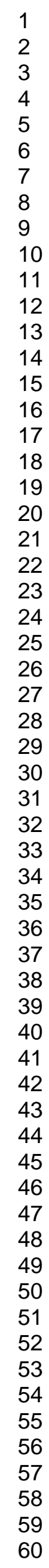

https://mc.manuscriptcentral.com/fla 
Asking for Action or Information? Crosslinguistic Comparison of Interrogative Functions in Early Child Cantonese and Mandarin

\author{
Hui Li ${ }^{\mathrm{a},{ }^{*}}$, Eileen Chin Mei Wong ${ }^{\mathrm{a}}$, Shek Kam Tse ${ }^{\mathrm{a}}$, Shing On Leung ${ }^{\mathrm{b}}$, Qianling Ye ${ }^{\mathrm{b}}$ \\ ${ }^{\text {a }}$ Faculty of Education, The University of Hong Kong, PRC \\ ${ }^{\mathrm{b}}$ Faculty of Education, University of Macau, PRC
}

\title{
Acknowledgements
}

This article is based on a project (RGC Ref No. 747109) funded to the first author by the Research Grants Council of the Government of the Hong Kong SAR. Thanks are given to all the participating children and their parents, Prof. A.H. Liu, Dr. Eva Chen, and the student research assistants. 


\begin{abstract}
Request for information (RfI) is believed to be the universally dominant function of young children's questioning, whereas request for action (RfA) has been reported to be the leading interrogative form used in early child Cantonese. The possibility of crosslinguistic variability prompts further research and comparison with additional languages. This study compared the interrogatives elicited from two early Chinese language corpora: Early Childhood Cantonese Corpus (ECCC) and Early Childhood Mandarin (ECMC). Altogether, 1,214 and 942 question types were elicited from ECCC and ECMC, respectively. Analyses indicated that: (1) All the interrogative functions identified in an earlier study of Cantonese were also observed in the early Mandarin interrrogatives; and (2) both RfA (49.9\%) and RfI (45.5\%) were the most frequently observed functions of early child Chinese interrogatives. This crosslinguistic evidence suggests that follow-up studies are needed to further explore the possible influences of language, culture and communication tasks on children's uses of interrogative forms.
\end{abstract}

\title{
Keywords
}

Interrogative function, early child Mandarin, early child Cantonese, corpus-based study 


\section{Introduction}

In a comprehensive monograph reviewing early question acquisition studies,

Chouinard (2007) proposed a relatively comprehensive taxonomy of interrogative functions: (1)

Information seeking questions requesting (a) isolated facts without causal component; (b)

explanations with a causal component; (2) Action-beseeching questions requesting (a)

attention, (b) action, (c) permission, or (d) play; and (3) Non-information-seeking questions

including (a) those addressed to a child/animal who cannot answer, and (b) all the questions

that cannot be classified into any category. Among these categories, information-seeking was

found to be the dominating function, accounting for $81 \%$ of child questions. This indicates

that the ability to ask questions to gather information constitutes a fundamental mechanism in

cognitive development. Chouinard (2007) termed such a mechanism the 'Information

Requesting Mechanism' (IRM), which allows children to obtain information they think they

need and to move their knowledge structures closer to adult-like states. Her findings indicated

that the components of the IRM are in place and are used by children under 30 months.

Although the IRM hypothesis has been examined in the context of a wide range of

Western languages, its validity has been explored less in Asian languages such as Chinese, and its universality is hence less certain (Maratsos, 2007). For instance, our previous study (Li et al., 2013) found that Request for Action (RfA) turned out to be the leading function (54\%) in early Cantonese interrogatives, although Request for Information (RfI) was still frequently used $(39 \%)$ in the free play context during peer conversations. To further examine the generality and 
applicability of the IRM hypothesis, this study will compare the interrogatives elicited from two early child Chinese language corpora: Early Childhood Cantonese Corpus (ECCC, Tse \& Li, 2011) and Early Childhood Mandarin (ECMC, Li \& Tse, 2011). Both Cantonese and Mandarin feature a very complicated system of questions (Gao, 2000; Matthew \& Yip, 1994, 2011), and this study will provide crosslinguistic evidence to test whether the leading RfA function in early Cantonese interrogative is also observed in early Mandarin interrogatives. The following questions guided the study:

1. What interrogative functions are present in early child Cantonese and Mandarin? Additionally, does the typology of Cantonese interrogative functions proposed by Tse and Li (2011) also apply to Mandarin Chinese?

2. Is Request for Action (RfA) a dominant function in Chinese young children's questioning?

In particular, we tested the following predictions:

H1. All the Cantonese interrogative functions suggested by Tse and Li (2011) could be found in early child Mandarin.

H2. Request for Action (RfA) would be the leading function (around 50\%) in early child Cantonese and Mandarin.

\author{
Method \\ The corpora
}


Two early child Chinese corpora were used in this investigation: the Early Child Cantonese Corpus (ECCC) (Tse \& Li, 2011) and the Early Child Mandarin Corpus (ECMC) (Li \& Tse, 2011).

The ECCC. This corpus consisted of the utterances produced by 492 Cantonese-speaking pre-schoolers as they participated in pairs in a 30-minute play task. They were randomly sampled from 68 preschools (58 kindergartens and 10 nurseries) located on Hong Kong. This sample represented children from three age groups (age 3:0, 4:0 and 5:0), with 82 boys and 82 girls in each age group. All the participants were native speakers of Cantonese, and their parents and teachers also spoke Cantonese at home and at preschool respectively. For more information about this corpus, please refer to Tse and Li (2011).

The ECMC. This corpus consisted of the utterances produced by 192 Mandarin-speaking preschoolers randomly sampled from 8 preschools located in the four districts of Beijing. This sample represented children from four age groups (age 2;0, 3;0, 4;0 and 5;0), with 24 boys and 24 girls in each age group. All the participants were native speakers of Mandarin, and their parents and teachers also spoke Mandarin at home and at preschool respectively.

Significant similarities rather than differences were found in the two corpora, implying a satisfactory level of comparability. The corpora were conducted by the same research team using the same research design, topic, communication task, protocol, and children`s toys. The only difference was that the ECMC had four age groups, with a younger age group (age $2 ; 0)$ 
which was not included in the ECCC. Therefore, we excluded 2-year-olds from the comparison and just focused on three age groups: age 3;0, 4;0 and 5;0, for a more valid and feasible comparison between the two corpora.

The communication task. The corpora shared the same communication task. A toy play area furnished with the same set of toys, including cooking materials, food and fruits, furniture and electrical appliances and hospital materials and vehicles, was set up in the classroom of participating kindergartens. Only one dyad of same age participants at a time was allowed to play in the room for 30 minutes, and their conversations were audio-recorded using an unobtrusive recorder. The researchers observed the activities during the free play sessions but did not make any interventions.

All audio-taped conversations were accessed by two research assistants using a digital audio player that allowed automatic rewinding for repeated playing at normal speed. The research assistants transcribed these conversations to a level of detail that captured all words and word fragments (including overlapping speech) audible to the ear, non-lexical fillers (e.g. ' $u h^{\prime}$ ), as well as other vocalizations (e.g. laughter). Other researchers then independently proofread the transcripts against the taped audio clips to ensure accuracy of transcription.

\section{Results}

Altogether, 1,214 and 942 question types were identified in the Early Child Cantonese Corpus (ECCC) and Early Child Mandarin Corpus (ECMC). The three-year age group 
produced all the 14 interrogative functions suggested by Tse and Li (2011), which could also be placed into the three major categories suggested by Chouinard (2007): (1) Information seeking (Request for Information, RfI), (2) Action-beseeching (Request for Action, RfA), and (3) Non-information-seeking. In contrast to Chouinard's (2007) findings, which showed information seeking as the dominant function of the interrogative behaviour of children, the interrogatives produced by the three-, four- and five year old Cantonese and Mandarin speakers in this study served the function of soliciting action $(49.4 \% ; 50.4 \%$ respectively) more often than seeking information (44.7\%; 46.5\% respectively) or other functions. As shown in Table 1 , the early childhood Cantonese and Mandarin data show exactly the same pattern of distribution of the four major interrogative functions. Also, the mean percentages for the interrogatives in the early childhood Cantonese and Mandarin data are $49.9 \%$ for RfA versus $45.5 \%$ for RfI (See Table 1), indicating that both RfA and RfI were the most frequently observed interrogative function among the young Cantonese and Mandarin speakers. This dual-function of early Chinese interrogative deserves further discussion and follow-up studies.

Insert Table 1 about here 


\section{Discussion}

As the first comparison ever made of interrogative functions in early child Cantonese and Mandarin, the study provides robust evidence about the development of Chinese interrogative functions to test the IRM hypothesis (Chouinard, 2007). Generally, all the pragmatic functions were found in early Chinese interrogatives. But the Information Requesting Mechanism (IRM) hypothesis is not supported by the present study. Instead, both RfA and RfI turned out to be the most frequently observed function of early child Cantonese and Mandarin interrogatives. This inconsistent finding regarding the RfI versus RfA controversy might be related to the differences in language, culture, and/or communication task (Li, et al., 2013).

Gardner (1989) and Chan (1995) asserted that education in the Chinese collectivist culture was often teacher-centred oriented, and students were encouraged to follow their teachers' instructions to the letter. In contrast, education in Western liberal cultures seems to emphasize the promotion of individual characteristics in children, such as exploration, creativity, sensitivity and independence. If such a postulation holds true, it seems reasonable for Cantonese- and Mandarin-speaking children to seek actions and confirmations, rather than information, more often than their Western peers. However, this assumption might also imply that Chinese cultural values and educational attitudes cultivate passive, dependent learners rather than active learners, an idea that requires further investigation and deliberation. 
Further, the outcomes of the present study may be a reflection of the social nature of the communicative tasks. The free play areas in this study were furnished with toys for boys as well as girls, and the participants were randomly paired and allowed to play in the room. During the play, the communicative tasks were basically social and interactive, allowing the participants collaboratively to engage in role play and to share or exchange interesting toys. This might in part explain why these Chinese-speaking preschoolers produced relatively more questions about actions than those requesting information. In this connection, the noteworthy difference between the dominating RfI pattern in English interrogatives (81\%, Chouinard, $2007)$ and the dual-function pattern $(\mathrm{RfA}+\mathrm{RfI})$ in Chinese $(49.9 \%+45.5 \%)$ might be due to the difference between the communicative tasks and the interlocutors in the two studies. In Chouinard's studies, the children were posing questions to parents or adults in different learning contexts, whereas the children in the present study were posing questions to their peers in a free play context. The different natures of the communicative contexts might have significantly influenced the purposes and functions of the interrogatives uttered by the two samples.

This crosslinguistic evidence suggests that the dominance of RfI might not be universal across languages; instead, it might vary between cultures and communication tasks. The early Chinese interrogatives during peer conversation, as shown in this study, feature a dual-function of requesting action and information. Nevertheless, follow-up studies are needed to further 
explore the relative influences of culture and communication task and their interactions.

There are advantages as well as disadvantages associated with the use of a corpus approach in this study. First of all, the two corpora followed the same design, protocol and coding system, which granted a comprehensive crosslinguistic comparison of early childhood Cantonese and Mandarin. Second, using the corpora as a primary source of evidence allowed us to test the crosslinguistic generality of Chouinard's findings. However, a major limitation of this corpus-based research is that the data only represented a sample of the entire language, not the target language in its totality. It is quite natural that corpus is likely to be skewed in its content and the extent to which it reflects the wider scenario of everyday use. Therefore, corpus linguists need to bear in mind that no particular corpus will contain information about all of the areas of language that they may want to explore (Tse \& Li, 2011). To minimize this eventuality, the two corpora could be more comprehensive and inclusive, and should be derived from a larger sample with data collected from more typical, everyday contexts. These might be the limitations of this study. Nevertheless, the study provides additional evidence that the patterns reported for early development of interrogatives in western languages may not be universal. Future research could address patterns in the input provided by caregivers as well as the influence of culturally-embedded assumptions about how and when young children are guided to use (or not to use) particular interrogative forms. 


\section{References}

Chan, Y. M. (1995). Chinese traditions and education: An overview. Education Today, 45(2), 20-25.

Chouinard, M. (2007). Children's questions: A mechanism for cognitive development. Monographs of the Society for Research in Child Development, 72(1, Serial No. 286).

Gao, M. C. F. (2000). Mandarin Chinese: An introduction. New York: Oxford University Press.

Gardner, H. (1989). To open minds: Chinese clues to the dilemma of contemporary education. New York: Basic Books.

Li, H., Tse, S. K., Wong, J. M. S., Wong, E. C. M., \& Leung, S. O. (2013). The development of interrogative forms and functions in early childhood Cantonese. First Language, 33(2), 168-181.

Li, H., \& Tse, S.K. (2011). The Early Child Mandarin Corpus. Hong Kong: The University of Hong Kong.

Maratsos, M. P. (2007). Commentary. In M.M.Chouinard, Children’s questions: A mechanism for cognitive development. Monographs of the Society for Research in Child Development, 72(1, Serial No. 286), pp.121-126.

Tse, S.K., \& Li, H. (2011). Early Child Cantonese: Facts and Implications. Volume 42 of Studies on Language Acquisition [SOLA] Series. Berlin, Germany: Gruyter Mouton. 
Table 1

Comparison of the Interrogative Functions produced by the Cantonese- and Mandarin-Speaking Preschoolers

\begin{tabular}{llll}
\hline Interrogative Functions & Cantonese & Mandarin & Mean \\
& $(\mathrm{n}=1214)$ & $(\mathrm{n}=942)$ & $(\mathrm{C}+\mathrm{M})$ \\
\hline Request for information & $543(44.7 \%)$ & $438(46.5 \%)$ & $981(45.5 \%)$ \\
Request for action & $600(49.4 \%)$ & $475(50.4 \%)$ & $1075(49.9 \%)$ \\
Emotional expression & $51(4.2 \%)$ & $9(1.0 \%)$ & $60(2.8 \%)$ \\
Egocentric speech & $20(1.7 \%)$ & $20(2.1 \%)$ & $40(1.9 \%)$ \\
\hline
\end{tabular}

